\title{
Precise single column resection and reconstruction with femoral head plus THR for malignant pelvic tumors
}

Xiaohui Niu*, Yongkun Yang, Qing Zhang, Yuan Li, Hairong Xu, Weifeng Liu Department of Orthopedic Oncology, Beijing Ji Shui Tan Hospital, Peking University, Beijing, People's Republic of China.

*Corresponding author

Xiaohui Niu MD

Professor, Director of Department of Orthopedic Oncology

Beijing Ji Shui Tan Hospital, Peking University, Beijing, China

\begin{abstract}
Background Some malignant pelvic tumor may affect the anterior or posterior column at the acetabula area. The postoperative recurrence and complication rate are high. Precise resection with safe surgical margin could cure the patients while saving healthier host bone for relative simple reconstruction. The purpose of this study is to evaluate the effect of precise single column resection and reconstruction with femoral head plus THR for malignant pelvic tumors.
\end{abstract}


Methods From 2007 to 2015, 19 patients with primary malignant tumors of the pelvis were enrolled in the study. All tumors were resected with safe surgical margins, which were proved by the postoperative specimen evaluation. Anterior column was involved in 17 cases and posterior column in 2 cases. Ten of 19 tumors were resected assisted by computer navigation. Femoral heads were used to reconstruct anterior or posterior column defects and fixed by screws; THR was used for the joint reconstruction. Oncologic outcome and function were evaluated by regular follow-up. Results The average follow-up time was 65.9 months (median 65.5, range 12-149) months. Surgical margins contained wide resection in 12 cases and marginal resection in 7 cases. The bony wide resection rate was $90 \%(9 / 10)$ in the navigation group and $77.8 \%(7 / 9)$ in free hand group respectively. One patient with Ewing's sarcoma died 14 months postoperative due to lung metastasis. There was only one case with chondrosarcoma was found recurrence in 61 months postoperatively, who was in the navigation group and having marginal margin resection. There was one prosthesis removed due to prosthesis infection (14 months postoperatively). There were another two patients with minor wound infection. The average MSTS function score was $25.1(17-29)$.

Conclusions The current treatment method is oncological safe and functional with less complications. Partial acetabulum can be reserved through precise plan of resection and reconstruction.

\section{Introduction}

Due to the complexity of the anatomical structure, surgical treatment of pelvic tumor is very difficult. The intraoperative risk and recurrence rate is high. More 
complications can be found postoperatively [1-3]. Some special malignant pelvic tumor may only affect the anterior or posterior column at the acetabula area. How to control the recurrence rate and apply effective reconstruction is difficult. In recent years, we have performed the study on the reconstruction of bone tumors involving the acetabulum by strict preoperative planning and precise single column resection with respect to the surgical safty, oncological outcome and prosthesis survivorship and function. .

\section{Methods}

All cases were retrospectively reviewed in our database and the operation time was from 2007 to 2015 . 19 cases were included according to the inclusion criteria: tumors were located in the anterior or posterior column of the acetabulum (zone II), also involving the obturator area (zone III); pathological confirmed primary pelvic malignant tumor; weight-bearing or stability of the acetabulum was affected and reconstruction was necessary; surgical resection of anterior column or posterior column and total hip joint replacement was performed. There were 9 males and 10 females. Mean age was 47.2 (24-64) years. Pathological diagnosis included 16 cases of chondrosarcoma (tumor grade: nine cases were grade 1, six cases with grade 2 and one case with grade 3), 1 case of undifferentiated pleomorphic sarcoma (UPS), 1 cases of Ewing's sarcoma and 1 case of solitary plasmacytoma. The lesion sites included 17 cases of anterior column and 2 cases of posterior column.

All cases were treated with tumor resection. The osteotomy line was designed to include a $1.5-\mathrm{cm}$ margin of normal bone from the edge of the tumor. Intra-operative computer navigation was randomly applied in the cases. Computer 
navigation aided surgery was performed in 10 cases. The CT and MRI images were fused in the navigation system (Stryker Orthomap 3D Navigation System). The accurate three-dimensional model of the tumor was showed and pre-operative resection planes and margins were designed (Figure 1). Tumor resection was performed intraoperative according to the preoperative plan (Figure 2-3). The autologous ipsilateral femoral head was shaped and implanted in the acetabular defect with the surfaces of cancellous bone contacted together. A few long screws helped to fix it stable. The reconstructed acetabular was then shaped and cemented total hip prosthesis replacement was performed (Figure 4). The postoperative specimen was cut and evaluated (Figure 3).

\section{Results}

The average amount of blood loss in navigation group and non-navigation group was $2000 \mathrm{ml}$ and $3200 \mathrm{ml}$, respectively $(\mathrm{p}=0.500)$. The average operation time in navigation group and non-navigation group was 298 and 351 minutes, respectively $(\mathrm{p}=0.455)$. The planned clear surgical margins were obtained in all cases. The bony wide resection (1.5-cm of normal bone from the edge of the tumor) rate was $90 \%$ $(9 / 10)$ in the navigation group and $77.8 \%(7 / 9)$ in free hand group, respectively $(\mathrm{p}=0.819)$.

The average follow-up time was 65.9 months (median 65.5, range 12-149) months. One patient with Ewing's sarcoma died of lung metastasis 14 months postoperative. One patient with chondrosarcoma had recurrence 61 months postoperative and semi pelvic amputation was performed. The recurrence rate in non-wide margin group and 
wide margin group was $14.3 \%(1 / 7)$ and $0(0 / 12)$, respectively $(p=0.251)$.

Two prosthesis failures occurred. One case was received joint fusion 14 months postoperative due to deep infection. Another patient was the recurrent case. There was no postoperative prosthesis dislocation and other prosthesis related complication. The 5 year overall survival rates were $100 \%$. The average survival time was 102.8 months. The average MSTS score was 25.1 (17-29).

\section{Discussion}

Surgical resection of malignant tumor in the acetabulum is difficult. The risk of operation and postoperative recurrence rate is high. A lot of reports [4-8] showed the recurrence rate of pelvic chondrosarcoma was about $18-45 \%$ and surgical margin was related to local recurrence significantly.

Our study showed only one local recurrence with chondrosarcoma. The surgical margin was marginal. Our study showed the recurrence rate was $14.3 \%(1 / 7)$ in nonwide cases and $0(0 / 12)$ in wide cases. Therefore, the premise of reducing recurrence is the safe surgical margin and accurate preoperative design and intraoperative performance. The sacrifice of large chunks of normal tissue can certainly obtain safe margin, but it also brings trouble in the reconstruction and increases the risks and complications after operation.

A number of studies [9-11] on the saddle prosthesis showed high complication rates. These included the destruction of iliac wing, prosthesis moving up, shortened limb length and prosthesis dislocation. The incidence rate of semi pelvic prosthesis reconstruction was up to $75 \%$ and the most common was wound related problems $[12,13]$. Reconstruction with massive allograft also has high risk. Ozaki et al [14] 
reported 22 cases of massive allograft reconstruction, but 2 cases of fracture and 8 cases of infection were found.

Total hip arthroplasty has certain advantages in the reconstruction of acetabular, but there are also some problems. Our study showed 2 prosthesis failures and one of them was due to tumor recurrence. This also suggested that the main reason of prosthesis failure was tumor recurrence. Another failure was caused by deep infection. Our results showed high 5 year prosthesis survival rate. The mean postoperative MSTS score was 25.1 with acceptable daily activity. All the patients were able to walk and take care of themselves. Our surgical resection and reconstruction method depends on good preoperative design. Through accurate preoperative resection design and safe removal of the tumor, good local control can be obtained. We also retained anterior or posterior column to create condition for stable reconstruction.

In conclusion, the current treatment method is oncological safe and functional with less complication. Partial acetabulum can be reserved through precise plan of resection and reconstruction.

\section{References}

[1] Reguerre Y, Martelli H, Rey A, et al. Local therapy is critical in localised pelvic rhabdomyosarcoma: experience of the International Society of Pediatric Oncology Malignant Mesenchymal Tumor (SIOP-MMT) committee. European Journal of Cancer, 2012, 48(13):2020-2027.

[2] Delloye C, Banse X, Brichard B, et al. Pelvic reconstruction with a structural pelvic allograft after resection of a malignant bone tumor. The Journal of bone and joint surgery. American Volume, 2007, 89(3):579-587.

[3] Mankin HJ, Hornicek FJ, Temple HT, et al. Malignant tumors of the pelvis: an outcome study. Clinical Orthopaedics and Related Research, 2004, 425:212-217.

[4] Ozaki T, Hillmann A, Lindner N, Blasius S, Winkelmann W. Chondrosarcoma of the pelvis. ClinOrthopRelat Res 1997;337:226-39. 
[5] Gitellis S, Bertoni F, Campanacci M. Chondrosarcoma of bone. The experience at InstitutoOrthopedico Rizzoli. J Bone Joint Surg 1981;63A:1248—62.

[6] Donati D, El Ghoneimy A, Bertoni F, Di Bella C, Mercuri M. Surgical treatment and outcome of conventional pelvic chondrosarcoma. J Bone Joint Surg Br 2005;87(11):1527—30.

[7] Pring ME, Weber KL, Unni KK, Sim FH. Chondrosarcoma of the pelvis.A review of sixty-four cases. J Bone Joint Surg Am 2001;83-A(11):1630 — 42.

[8] Fiorenza F, Abudu A, Grimer RJ, Carter SR, Tillman RM, Ayoub K, et al. Risk factors for survival and local control in chondrosarcoma of bone. J Bone Joint Surg Br 2002;84(1): 93 —9.

[9] L. R. Menendez, E. R. Ahlmann, Y. Falkinstein, and D. C. Allison, "Periacetabular reconstruction with a newendoprosthesis," Clinical Orthopaedics and Related Research, vol. 467, no. 11, pp. 2831-2837, 2009.

[10] Aljassir F, Beadel GP, Turcotte RE, Griffin AM, Bell RS, Wunder JS, Isler MH. Outcome after pelvic sarcoma resection reconstructed with saddle prosthesis. ClinOrthopRelat Res. 2005;438:36-41.

[11] Cottias P, Jeanrot C, Vinh TS, Tomeno B, Anract P. Complications and functional evaluation of 17 saddle prostheses for resection of periacetabular tumors. J SurgOncol. 2001;78:90-100.

[12]Witte D, Bernd L, Bruns J, Gosheger G, Hardes J, Hartwig E, et al. Limb-salvage reconstruction with MUTARS hemipelvicendoprosthesis: a prospective multicenter study. Eur J SurgOncol. 2009;35:1318-25.

[13]Ji T, Guo W, Yang RL, Tang XD, Wang YF. Modular hemipelvicendoprosthesis reconstruction experience in 100 patients with mid-term follow-up results. Eur J SurgOncol. 2013;39:53-60.

[14] T. Ozaki, A. Hillmann, D. Bettin, et al."High complication rates with pelvic allografts. Experience of 22 sarcoma resections," ActaOrthop Scand. 1996; 67(4): 333-8. 


\section{Figures}

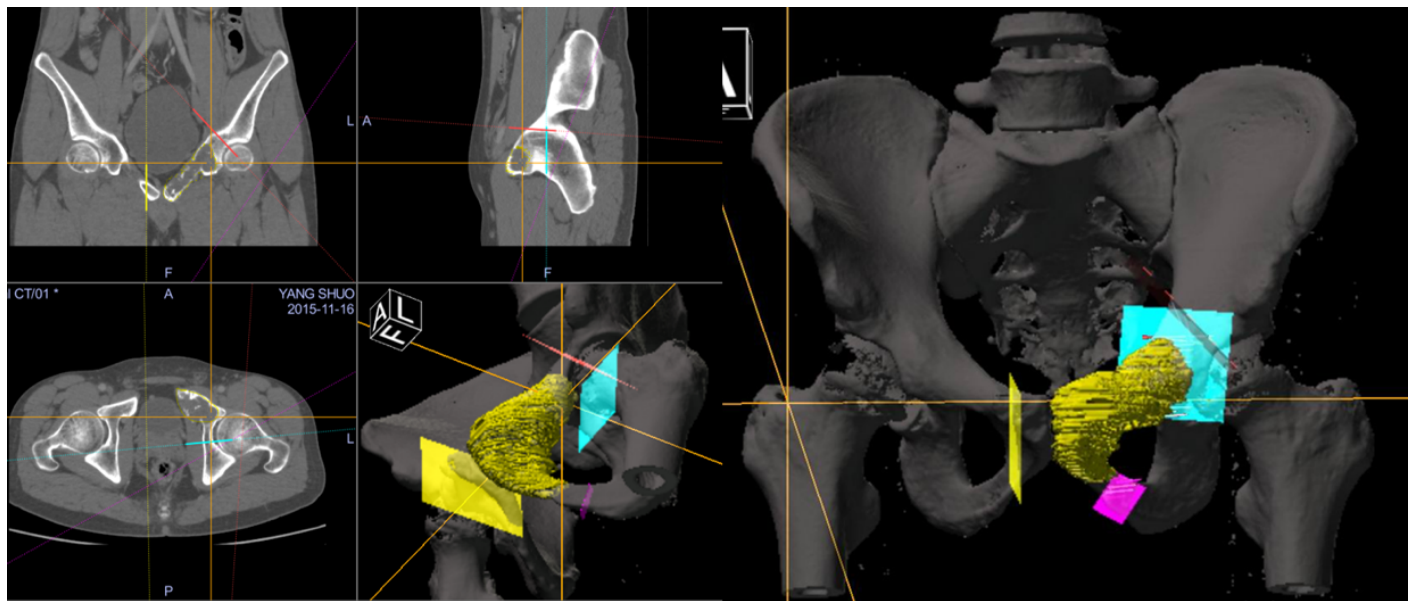

Figure 1 The tumor margin and resection plan was designed in navigation system. Tumor range was described as yellow area and the osteotomy planes were designed by virtual planes with different colors. Different cross sections and three-dimensional images showed the resection design (1.5- $\mathrm{cm}$ from the edge of the tumor). The anterior column of acetabulum could be resected safely and posterior could be reserved according to the preoperative plan.

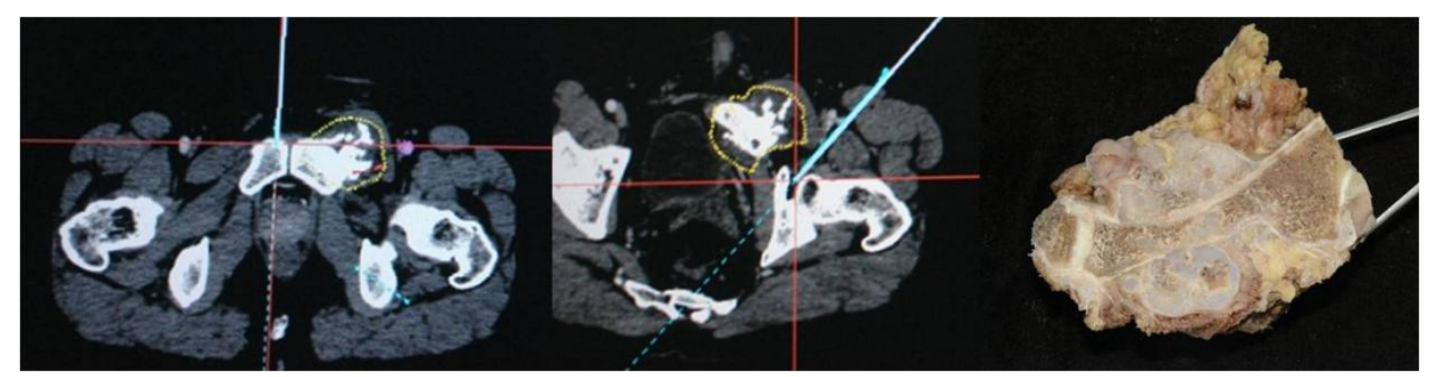

Figure 2 The precise tumor resection was performed under the direction of navigation system (intraoperative images of navigation). The postoperative specimen was cut and evaluated. 


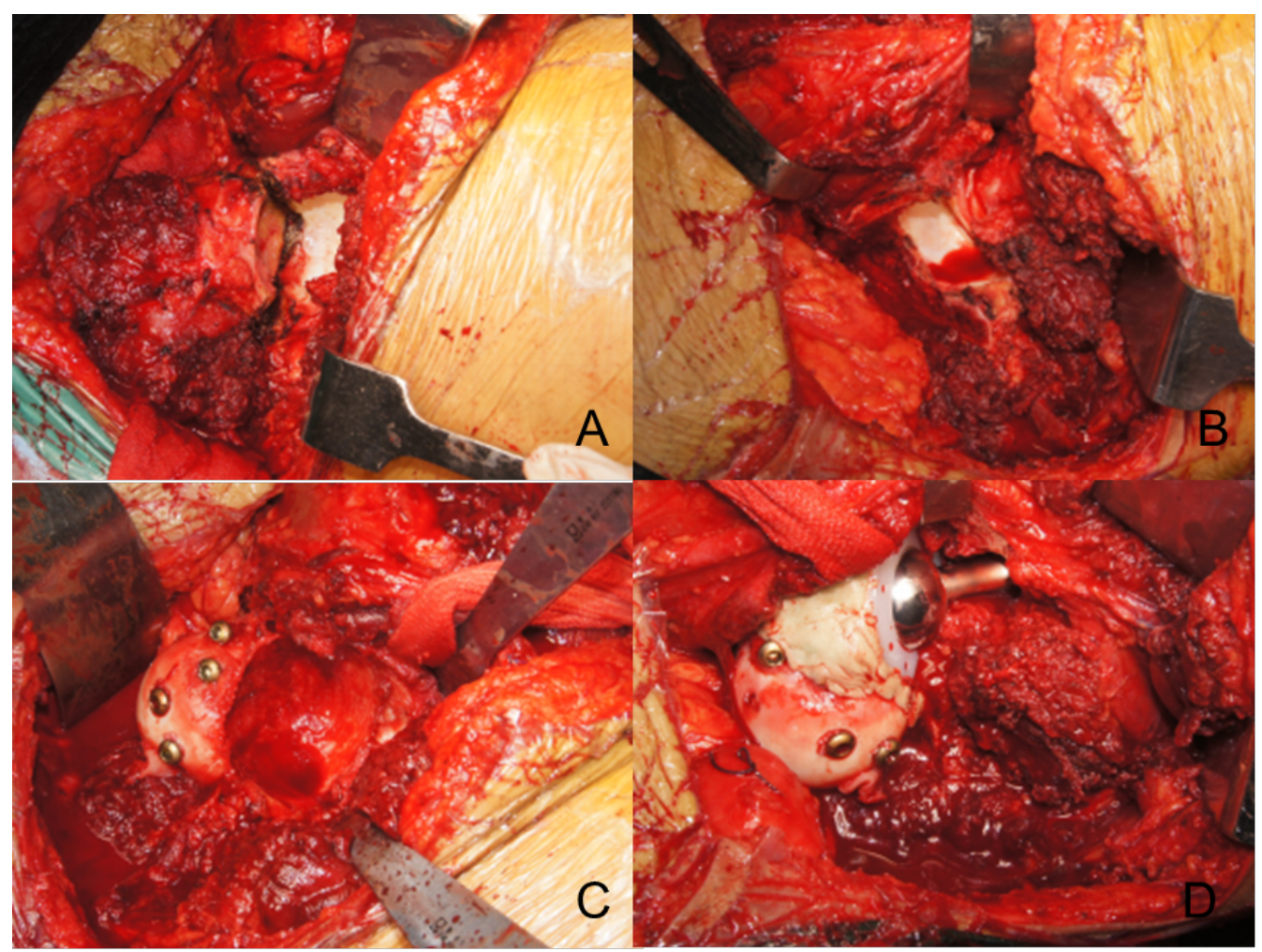

Figure 3 The precise single column resection and reconstruction with femoral head plus THR. A. The bone resection line was marked with the direction of intraoperative navigation. B. The precise single column resection was then performed. $\mathrm{C}$. The autologous ipsilateral femoral head was shaped and implanted in the acetabular defect. D. The cemented total hip prosthesis replacement was performed. 


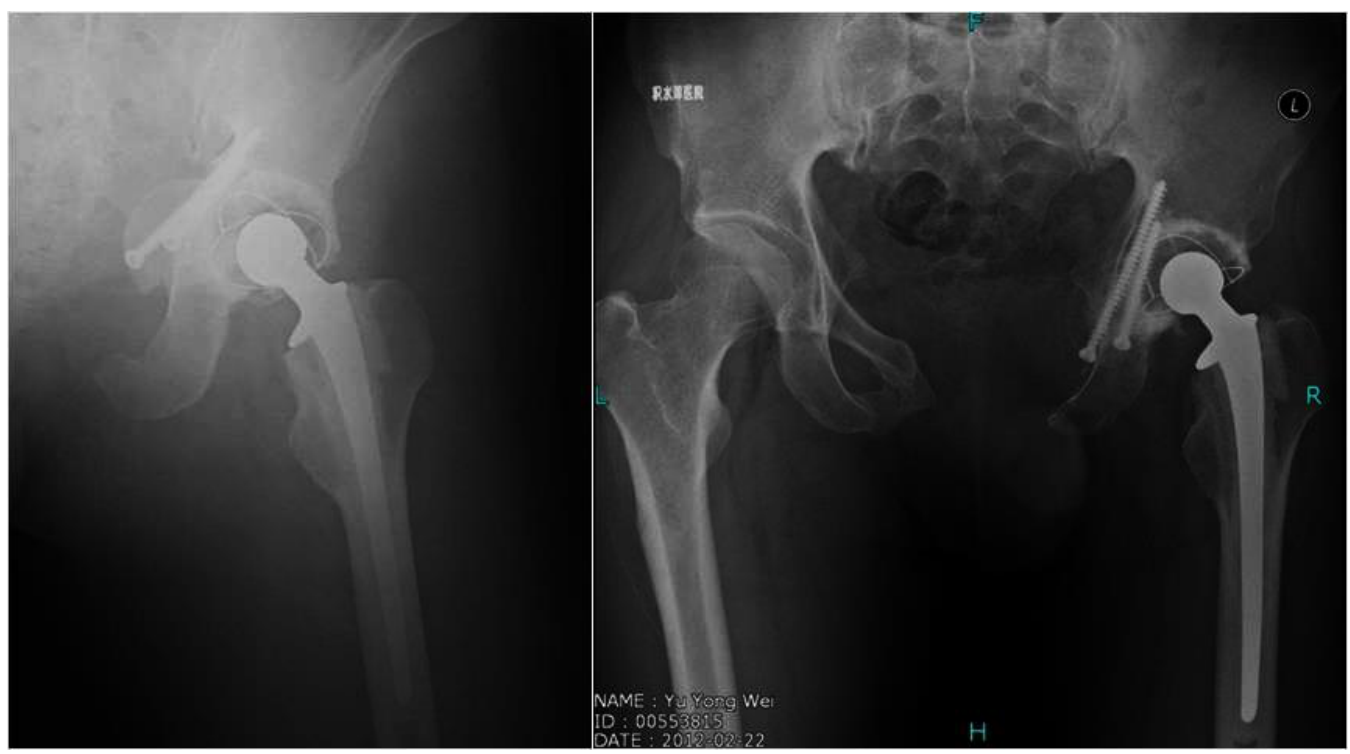

Figure 4 The radiography postoperative and 50 months postoperative 